\title{
Ajustamento para Heterogeneidade de Variância da Produção de Leite de Vacas da Raça Holandesa no Estado de Minas Gerais
}

\author{
Nilson Milagres Teixeira' ${ }^{1}$ Ary Ferreira de Freitas ${ }^{1}$, William José Ferreira ${ }^{2}$, Marcus Cordeiro Durães ${ }^{1}$, \\ Ricardo Bertola Barra ${ }^{3}$
}

\begin{abstract}
RESUMO - Utilizou-se para avaliação genética de vacas da raça Holandesa no Estado de Minas Gerais um procedimento de ajustamento para heterogeneidade de variância entre rebanhos e ao longo do tempo, com base na variância para produção de leite em cada subclasse rebanho-ano. As variâncias fenotípicas duplicaram com o aumento da média de produção da subclasse de 4.000 para 10.000 $\mathrm{kg}$ e cresceram, também, com o número de animais na subclasse. As produções foram padronizadas para uma variância comum e os valores genéticos obtidos antes e após o ajustamento, comparados. As variações das Capacidades Previstas de Transmissão (PTAs) e ordem dos touros foram pequenas. As correlações de ordem entre PTAs de vacas elite foram baixas até negativas, sendo antes originadas de rebanhos com maior variabilidade e, após o ajustamento, selecionadas em todas as classes de variabilidade. Com o ajustamento para heterogeneidade da variância, houve aumento de $12 \mathrm{~kg}$ /ano na tendência genética estimada para a produção de leite. A implementação do ajustamento para heterogeneidade de variância nas avaliações genéticas pode contribuir para a seleção mais precisa de vacas elite no Estado e poderá aumentar a taxa de ganho genético pela seleção de fêmeas.
\end{abstract}

Palavras-chave: avaliação genética, gado de leite, heterogeneidade de variância, produção de leite

\section{Adjustment for Heterogeneity of Variance for Milk Yield of Holstein Cows in Minas Gerais State}

\begin{abstract}
A method was used to account for heterogeneous phenotypic variance for milk yield across herds and over time based on variance for each herd-year class in genetic evaluation of Minas Gerais State Holstein. Phenotypic variances increased twice as within subclass averages increased from 4,000 to $10,000 \mathrm{~kg}$ and also with increases in subclass size. Data were standardized to a common variance and the breeding values obtained before and after adjustment were compared. Small variations of Predicted Transmitting Abilities (PTAs) and ordering for sires were found. Rank correlations between PTAs of elite cows were small to negatives. Before adjustment for heterogeneity the elite cows originated from herds with large variability and after they were selected in all variability classes. Estimated genetic trend for milk increased by $12 \mathrm{~kg} /$ year after adjustment. The implementation of adjustment for heterogeneity of variance in genetic evaluation should increase the reliability of selection among top cows in the State and may increase the rate of genetic gain from female selection.
\end{abstract}

Key Words: dairy cattle, genetic evaluation, heterogeneous variances, milk yield

\section{Introdução}

Nas avaliações genéticas em gado de leite pressupõe-se a utilização de um modelo estatístico que melhor se ajusta aos dados de desempenho, isto é, que contemple o maior número possível de fatores que interferem na variação da produção, possibilitando comparações entre os animais com eliminação tanto quanto possível de diferenças não-genéticas. Além disso, o modelo deve ser apropriado à estrutura da população de onde os dados são originários, contemplar grupos contemporâneos com relação a manejo, grupos genéticos e diferenças de variação das características entre rebanhos.
Atualmente, no sistema de avaliação usado na raça Holandesa no Estado de Minas Gerais, assumese homogeneidade das variâncias genética e residual para todos os ambientes. Esta pressuposição pode não ser verdadeira quando as condições ambientes forem heterogêneas ou quando os desempenhos são obtidos durante um longo período de tempo. Em vários estudos (Boldman \& Freeman, 1990; Dong \& Mao, 1990; Meuwissen \& Van Der Werf, 1993; Van Der Werf et al., 1994) variâncias genética, residual e de meio permanente foram heterogêneas para características produtivas. Entre as fontes de variância heterogênea, provavelmente, a mais importante seja

\footnotetext{
${ }^{1}$ Pesquisadores da Embrapa Gado de Leite e bolsistas do CNPq - Rua Eugênio do Nascimento, 610, Dom Bosco, CEP: $36038-330$ Juiz de Fora - MG. E.mail: nilson@cnpgl.embrapa.br

${ }^{2}$ Estudante de Doutorado da UFV e Bolsista do CNPq. Depto. de Zootecnia: Av. PH Rolffs s/n, Viçosa-MG - CEP: $36570-000$.

${ }^{3}$ Técnico da Associação de Criadores de Gado Holandês de Minas Gerais - ACGHMG.
} 
o aumento da variância fenotípica com melhoria do nível de produção como um efeito de escala (RobertGranié et al., 1999). Em um mesmo ano, a variância fenotípica pode variar de um rebanho para outro, particularmente com o nível de produção e práticas de manejo. Diferenças entre rebanhos quanto à variação genética e heritabilidade já foram relatadas, as quais aumentaram com a produção e a variância fenotípica do rebanho. Esta tendência, contudo, não foi observada nos poucos trabalhos na raça Holandesa no Brasil, em que os valores da heritabilidade encontrados foram maiores para rebanhos de níveis médios de produção e desvio-padrão (Ramos et al., 1996; Torres et al., 1999). Outros pesquisadores, entretanto, não encontraram relação entre produção e heritabilidade (Mirande \& Van Vleck, 1985).

Se heterogeneidade de variância for ignorada, corre-se o risco de ordenação incorreta dos animais por meio de seus valores genéticos e, conseqüentemente, o progresso genético pode ser afetado. Se, entretanto, as filhas dos touros estiverem distribuídas, aleatoriamente, entre rebanhos de baixa e alta variabilidade, espera-se que a ordem dos touros não seja influenciada. Porém, a ordem das vacas poderá ser influenciada e aquelas que produzem em rebanhos com maior variabilidade poderão ser superavaliadas (Robert-Granié et al., 1999).

Vários métodos para correção para heterogeneidade da variância já foram sugeridos, dentre eles, transformação de escala dos dados por meio de desvio-padrão residual ou fenotípico (Hill, 1984; Weigel \& Gianola, 1992), enfoque de características múltiplas (Henderson, 1984; Gianola, 1986) e pré-ajustamento para variância fenotípica dentro de rebanhos (Wiggans \& Van Raden, 1991; Van Der Werf et al., 1994).

Valência et al. (1996), no Brasil, trabalhando com os dados de produção distribuídos entre três níveis, observaram que as transformações logarítmicas e raiz quadrada não estabilizaram os componentes de variância de touros, vacas e do resíduo. Torres et al. (1999) também constataram que as transformações dos dados de produção não eliminaram a heterogeneidade de variância entre os rebanhos pertencentes às diferentes classes de desvio-padrão fenotípico.

O objetivo deste trabalho foi determinar a magnitude da heterogeneidade da variância da produção de leite dentro de rebanho e o efeito de ajustamento para a mesma sobre os valores genéticos em rebanhos da raça Holandesa no Estado de Minas Gerais.

\section{Material e Métodos}

Os dados usados fazem parte dos arquivos da Associação de Criadores de Gado Holandês de Minas Gerais. Duas estações de parto foram consideradas: outubro a março e abril a setembro. Na edição dos dados, as classes rebanho-ano-estação com menos de dez lactações foram eliminadas. As produções de leite até 305 dias de 25.688 vacas com partos entre 1988 e 1999 foram pré-ajustadas para idade e estação de parto por meio de fatores multiplicativos, conforme Durães et al. (1999).

As variâncias das produções podem variar dentro de rebanho e entre anos para um determinado rebanho. A variação entre os anos ocorre porque as produções tendem a aumentar ao longo dos anos, e espera-se que as variâncias aumentem com a produção. Estimaram-se variâncias para cada subclasse rebanho-ano, por meio de:

$$
\hat{\sigma}_{i j}^{2}=\frac{\sum_{j=1}^{n_{i}}\left(y_{i j k}-\bar{y}_{i j}\right)^{2}}{n_{i j}-1}
$$

em que: $\hat{\sigma}_{i j}^{2}=$ variância estimada dentro de rebanhoano $\mathrm{ij} ; \mathrm{y}_{\mathrm{ijk}}=$ produção do animal $\mathrm{k}$ no rebanho-ano ij; $\mathrm{n}_{\mathrm{ij}}=$ número de produções no rebanho-ano ij;

Em uma análise preliminar ajustou-se o modelo linear fixo seguinte para identificar fontes de variação dos desvios-padrão fenotípicos dentro de cada subclasse rebanho-ano:

$$
\begin{aligned}
& \mathrm{D}_{\mathrm{ijklmno}}=\mathrm{Ano}_{\mathrm{i}}+\mathrm{Reb}_{\mathrm{j}}+\mathrm{Mês}_{\mathrm{k}}+\mathrm{CR}_{\mathrm{l}}+ \\
& \text { CLASNOB }_{\mathrm{m}}+\mathrm{CLASLEI}_{\mathrm{n}}+\mathrm{e}_{\mathrm{ijjlmno}}
\end{aligned}
$$

em que: $\mathrm{D}_{\mathrm{ijklmno}}=$ desvio-padrão fenotípico da produção de leite até 305 dias ajustada para idade e estação no ano i, rebanho j, mês k, grupo genético 1, classe de número de observação $\mathrm{m}$, classe de produção média de leite $\mathrm{n} ; \mathrm{Ano}_{\mathrm{i}}=$ efeito fixo de ano de parto i $(\mathrm{i}=$ $1988, \ldots, 1999) ; R^{2} b_{j}=$ efeito fixo de rebanho $\mathrm{j}(\mathrm{j}=$ $1, \ldots, 385) ;$ Mês $_{\mathrm{k}}=$ efeito fixo de mês de parto $\mathrm{k}(\mathrm{k}=$ $1, \ldots, 12) ; \mathrm{CR}_{1}=$ efeito fixo de grupamento genético 1 $(1=1, \ldots, 6) ;$ CLASNOB $_{m}=$ efeito fixo de classe de número de observações $\mathrm{m}(\mathrm{m}=1, \ldots, 6)$; CLASLEI $_{\mathrm{n}}$ $=$ efeito fixo de classe de produção média de leite $\mathrm{n}$ $(\mathrm{n}=1, \ldots, 8) ; \mathrm{e}_{\mathrm{ijk} \text { lmno }}=$ efeito aleatório de erro.

Uma vez que o número de lactações em cada subclasse rebanho-ano pode ser pequeno, os erros de amostragem das estimativas das variâncias podem ser grandes. Foram então estimadas variâncias pon- 
deradas dentro de rebanho-ano $\left(\hat{\sigma}^{2}{ }_{\text {wij }}\right)$, com menor erro de amostragem, combinando-se informação de anos adjacentes, isto é, a variância dentro de ano e as de rebanho-anos adjacentes conforme procedimento sugerido por Wiggans \& Van Raden (1991) e também usado por Van Der Werf et al. (1994). Os pesos para a ponderação das diferentes fontes de informação foram os mesmos de Wiggans \& Van Raden (1991). As produções foram, em seguida, padronizadas por meio de:

$$
y^{\prime}{ }_{i j k}=\left(y_{i j k}-\mu_{\text {RAEij }}\right) / \hat{\sigma}_{w i j}
$$

em que: $y^{\prime}{ }_{i j k}=$ produção padronizada; $\mu_{\mathrm{RAEijk}}=$ média de produção da classe $\mathrm{RA}_{\mathrm{ij}}$ (rebanho-ano) à qual pertence a produção $\mathrm{k}$.

As produções padronizadas foram, então, expressas na forma original usando-se como um valor constante a média geral das variâncias dentro de rebanho-ano. Para se obterem os valores genéticos, utilizou-se um modelo linear misto (modelo animal) contemplando os efeitos fixos de idade ao parto, rebanho, ano e estação de parto, grupos genéticos (PO e PC) e os efeitos aleatórios genético aditivo de animal e de meio ambiente permanente. O sistema MTDFREML (Boldman et al., 1995) foi usado.

Critérios para verificação de efeitos do ajustamento para heterogeneidade da variância foram: 1) correlações de ordem para touros e vacas; 2) mudanças de vacas elite entre classes de desviopadrão; 3) tendência genética na população de vacas.

\section{Resultados e Discussão}

Na Tabela 1 encontram-se número de subclasses rebanho-ano, número de lactações, médias das produções e dos desvios-padrão dentro das subclasses por ano e mês de parto e grupo genético. Houve aumento significativo da média dos desvios-padrão de 1988 até 1997, fato ocorrido provavelmente devido ao aumento na produção no decorrer do período. As mudanças dos desvios-padrão com o mês de parto não foram significativas. À medida que os animais passaram a ser mais controlados quanto aos seus pedigrees, constatou-se maior produção de leite e aumento da média dos desvios-padrão, concordando com Weigel et al. (1993), que verificaram variâncias residuais para características produtivas maiores em rebanhos registrados. Observou-se aumento de 100\% nas médias dos desvios-padrão entre os níveis extremos de produção (Tabela 2), indicando ser o nível de produção o principal fator de variabilidade dos rebanhos. Houve, também, aumento significativo na mé- dia dos desvios-padrão com o número de animais do rebanho, concordando com Weigel et al. (1993), os quais encontraram variâncias residuais maiores para produção de leite em rebanhos maiores.

Pela Tabela 3 e Figura 1, observam-se mudanças nas Capacidades Previstas de Transmissão (PTAs) dos touros e das vacas com o ajustamento para heterogeneidade da variância. As correlações de ordem entre PTAs obtidas antes e após o ajustamento para heterogeneidade, para todos os animais avaliados foram altas, respectivamente, 0,950 e 0,976 para touros e vacas. Quando os touros apresentavam PTA com maior confiabilidade e possuíam filhas em muitos rebanhos não foram afetados pela correção para heterogeneidade da variância. Entretanto, quando possuíam filhas em poucos rebanhos, as diferenças de ordem foram mais pronunciadas. Como constatado por Hill (1984), Wiggans \& Van Raden (1991), o ajustamento apresentou efeitos sobre os valores genéticos das vacas elite. As correlações foram muito menores até negativa para as mesmas, indicando a ocorrência de um importante reordenamento à medida em que se reduziu a amostra. Na Tabela 3 tem-se também o número de novos animais entre os elite. Quando se ajustou para heterogeneidade, aproximadamente $40 \%$ das vacas elite foram substituídas. Como relatado também por Wiggans \& Van Raden (1991), a origem das vacas elite foi também afetada (Tabela 4). Antes originavam-se de rebanhos com maior variabilidade e com o ajustamento houve uma troca de vacas destes para rebanhos de variabilidade média. Rebanhos com maior variabilidade contribuíram com 35 das 100 melhores vacas quando não houve correção para heterogeneidade e 16 quando houve correção. Proporcionalmente, rebanhos com maior variabilidade mantiveram a contribuição dos rebanhos com baixa variabilidade, uma vez que alta variabilidade estava associada a um alto nível de produção e a um alto nível genético médio.

Como já foi sugerido (Winkelman \& Schaeffer, 1988; Boldman \& Freeman, 1990), o efeito do ajustamento para heterogeneidade da variância sobre os valores genéticos dos touros foi pequeno. Entretanto, foram significativas as mudanças nos valores genéticos de vacas, sugerindo que possivelmente as PTAs de vacas antes do ajustamento estivessem sujeitas a vícios, devido à heterogeneidade da variância. Estas mudanças eram esperadas, uma vez que as produções de uma vaca ocorrem, na sua maioria, em um mesmo rebanho, pelo que a sua PTA está mais 
Tabela 1 - Número de subclasses rebanho-ano (RA) com pelo menos três observações, número de lactações, médias da produção e dos desvios padrão para cada classe RA por ano de parto, mês e grupo genético

Table 1 - Number of subclasses for herd-year (RA) with at least three observations, number of records, milk yield and standard deviation averages within RA by year and month of calving and genetic group

\begin{tabular}{|c|c|c|c|c|}
\hline $\begin{array}{l}\text { Efeito e nível } \\
\text { Effect and level }\end{array}$ & $\begin{array}{c}\mathrm{N}^{\circ} \text { Subclasses }(\mathrm{RA}) \\
\text { Subclasses }\left(N^{\circ}\right)\end{array}$ & $\begin{array}{c}\text { Lactações } \\
\text { Lactations }\left(N^{\circ}\right)\end{array}$ & $\begin{array}{l}\text { Média de produção }(\mathrm{kg}) \\
\text { Average yield }\end{array}$ & $\begin{array}{l}\text { Média desvio-padrão (kg) } \\
\text { Average standard deviation }\end{array}$ \\
\hline \multicolumn{5}{|l|}{$\begin{array}{l}\text { Ano de parto } \\
\text { Calving year }\end{array}$} \\
\hline 1988 & 23 & 496 & 6.861 & $1.198,16$ \\
\hline 1989 & 34 & 872 & 6.750 & $1.274,94$ \\
\hline 1990 & 90 & 3.347 & 6.792 & $1.234,57$ \\
\hline 1991 & 115 & 4.408 & 6.891 & $1.266,38$ \\
\hline 1992 & 129 & 4.580 & 6.864 & $1.280,30$ \\
\hline 1993 & 140 & 4.958 & 6.938 & $1.289,63$ \\
\hline 1994 & 155 & 5.997 & 6.979 & $1.313,77$ \\
\hline 1995 & 170 & 6.318 & 7.027 & $1.338,22$ \\
\hline 1996 & 191 & 6.631 & 7.037 & $1.337,55$ \\
\hline 1997 & 185 & 6.986 & 7.064 & $1.323,19$ \\
\hline 1998 & 184 & 7.082 & 7.065 & $1.357,82$ \\
\hline 1999 & 148 & 6.358 & 7.178 & $1.521,97$ \\
\hline \multicolumn{5}{|l|}{ Mês de parto } \\
\hline \multicolumn{5}{|l|}{ Month of calving } \\
\hline 1 & 125 & 4.087 & 6.910 & $1.309,34$ \\
\hline 2 & 100 & 4.305 & 6.925 & $1.312,06$ \\
\hline 3 & 145 & 5.915 & 6.984 & $1.316,02$ \\
\hline 4 & 177 & 6.120 & 6.937 & $1.316,39$ \\
\hline 5 & 164 & 6.232 & 7.014 & $1.308,67$ \\
\hline 6 & 148 & 5.704 & 7.021 & $1.307,66$ \\
\hline 7 & 132 & 5.558 & 6.999 & $1.309,49$ \\
\hline 8 & 126 & 5.182 & 7.104 & $1.303,66$ \\
\hline 9 & 97 & 4.216 & 7.050 & $1.313,62$ \\
\hline 10 & 118 & 3.708 & 6.838 & $1.319,38$ \\
\hline 11 & 104 & 3.468 & 6.785 & $1.309,83$ \\
\hline 12 & 128 & 3.538 & 6.882 & $1.310,40$ \\
\hline \multicolumn{5}{|l|}{ Grupo genético } \\
\hline \multicolumn{5}{|l|}{ Genetic group } \\
\hline $31 / 32$ & 285 & 10.583 & 6.706 & $1.305,49$ \\
\hline $\mathrm{GCl}$ & 70 & 8.726 & 6.895 & $1.308,31$ \\
\hline GC2 & 63 & 6.924 & 6.991 & $1.313,00$ \\
\hline GC3 & 28 & 4.849 & 7.021 & $1.318,17$ \\
\hline GC4 & 30 & 5.011 & 7.020 & $1.314,04$ \\
\hline $\mathrm{PO}$ & 1.088 & 21.940 & 7.090 & $1.309,25$ \\
\hline
\end{tabular}


Ajustamento para Heterogeneidade de Variância da Produção de Leite de Vacas da Raça Holandesa...

Tabela 2 - Número de subclasses rebanho-ano (RA), número de lactações, médias da produção e dos desvios-padrão para cada classe RA por número de vacas (NºBS) e produção media

Table 2 - Number of herd-year subclasses (RA), number of records, milk yield and standard deviations averages within RA by number of cows (NO.OBS) and production average

\begin{tabular}{|c|c|c|c|c|}
\hline $\begin{array}{l}\text { Efeito e nível } \\
\text { Effect and level }\end{array}$ & $\begin{array}{c}\mathrm{N}^{\circ} \text { Subclasses }(\mathrm{RA}) \\
\text { Subclasses }\left(N^{\circ}\right)\end{array}$ & $\begin{array}{c}\text { Lactações } \\
\text { Lactations }\left(N^{\circ}\right)\end{array}$ & $\begin{array}{c}\text { Média de produção }(\mathrm{kg}) \\
\text { Average yield }\end{array}$ & $\begin{array}{l}\text { Média desvio-padrão }(\mathrm{kg}) \\
\text { Average standard deviation }\end{array}$ \\
\hline \multicolumn{5}{|l|}{$\mathrm{N}^{\circ} \mathrm{OBS}$} \\
\hline $\begin{array}{l}<15 \\
15-29 \\
30-59 \\
60-99 \\
100-173 \\
\geq 174\end{array}$ & $\begin{array}{r}263 \\
561 \\
475 \\
215 \\
45 \\
5\end{array}$ & $\begin{array}{r}3.164 \\
12.000 \\
20.043 \\
16.401 \\
5.377 \\
1.048\end{array}$ & $\begin{array}{l}6.867 \\
6.908 \\
6.942 \\
6.909 \\
6.944 \\
7.154\end{array}$ & $\begin{array}{l}1.275,13 \\
1.270,95 \\
1.301,93 \\
1.289,54 \\
1.331,38 \\
1.399,32\end{array}$ \\
\hline \multicolumn{5}{|c|}{$\begin{array}{l}\text { Produção média } \\
\text { Average yield (kg) }\end{array}$} \\
\hline $\begin{array}{l}<4.000 \\
4.000-4.999 \\
5.000-5.999 \\
6.000-6.999 \\
7.000-7.999 \\
8.000-8.999 \\
9.000-9.999 \\
\geq 10.000\end{array}$ & $\begin{array}{r}57 \\
208 \\
374 \\
363 \\
284 \\
186 \\
75 \\
17\end{array}$ & $\begin{array}{r}1.448 \\
6.340 \\
12.435 \\
13.816 \\
11.685 \\
7.894 \\
3.737 \\
678\end{array}$ & $\begin{array}{r}4.077 \\
4.810 \\
5.675 \\
6.479 \\
7.269 \\
8.161 \\
8.958 \\
10.201\end{array}$ & $\begin{array}{r}882,85 \\
1.046,28 \\
1.157,88 \\
1.306,33 \\
1.360,61 \\
1.428,08 \\
1.510,31 \\
1.798,68\end{array}$ \\
\hline
\end{tabular}

Tabela 3 - Comparação das PTAs de touros e vacas com produções de leite antes e após ajustamento para heterogeneidade da variância dentro da subclasse rebanho-ano

Table 3 - Comparison of sire and cow PTA for milk yield before and after adjustment for heterogeneous phenotypic variance within herdyear subclass

\begin{tabular}{|c|c|c|c|c|}
\hline \multirow[b]{2}{*}{$\begin{array}{l}\text { Critério } \\
\text { Criterium }\end{array}$} & \multicolumn{2}{|c|}{$\begin{array}{l}\text { Touros } \\
\text { Sires }\end{array}$} & \multicolumn{2}{|c|}{$\begin{array}{l}\text { Vacas } \\
\text { Cows }\end{array}$} \\
\hline & $\begin{array}{c}\text { Sem ajuste } \\
\text { W/O adjustment }\end{array}$ & $\begin{array}{c}\text { Com ajuste } \\
\text { With adjustment }\end{array}$ & $\begin{array}{c}\text { Sem ajuste } \\
\text { W/O adjustment }\end{array}$ & $\begin{array}{c}\text { Com ajuste } \\
\text { With adjustment }\end{array}$ \\
\hline $\begin{array}{l}\text { Média } \\
\text { Average }\end{array}$ & 4,73 & 14,21 & 30,54 & 56,56 \\
\hline $\begin{array}{l}\text { Desvio-padrão } \\
\text { Standard deviation }\end{array}$ & 133,09 & 181,81 & 182,54 & 259,88 \\
\hline $\begin{array}{l}\text { Valormáximo } \\
\text { Maximum value }\end{array}$ & 976,90 & $1.367,90$ & $1.257,13$ & $1.265,19$ \\
\hline $\begin{array}{l}\text { Valor mínimo } \\
\text { Minimum value }\end{array}$ & $-666,34$ & $-844,08$ & $-794,72$ & $-877,69$ \\
\hline $\begin{array}{l}\text { Diferença máxima } \\
\text { Maximum difference }\end{array}$ & \multicolumn{2}{|c|}{352,23} & \multicolumn{2}{|c|}{94,57} \\
\hline $\begin{array}{l}\mathrm{N}^{\circ} \text { animais } \\
\text { Animals }\left(n^{\circ}\right)\end{array}$ & \multicolumn{2}{|c|}{2.799} & \multicolumn{2}{|c|}{31.735} \\
\hline \multicolumn{5}{|l|}{$\begin{array}{l}\text { Correlação de ordem } \\
\text { Rank correlation }\end{array}$} \\
\hline $\begin{array}{l}\text { Todos } \\
\text { All animals }\end{array}$ & \multicolumn{2}{|c|}{0,950} & \multicolumn{2}{|c|}{0,976} \\
\hline $\begin{array}{l}\text { Melhores } 100 \\
\text { Top } 100\end{array}$ & \multicolumn{2}{|c|}{0,826} & \multicolumn{2}{|c|}{0,420} \\
\hline $\begin{array}{l}\text { Melhores } 25 \\
\text { Top } 25\end{array}$ & \multicolumn{2}{|c|}{0,707} & \multicolumn{2}{|c|}{0,384} \\
\hline Melhores 10 (Top 10) & \multicolumn{2}{|c|}{0,794} & \multicolumn{2}{|c|}{$-0,479$} \\
\hline \multicolumn{5}{|l|}{$\begin{array}{l}\text { Novos animais } \\
\text { New animals }\end{array}$} \\
\hline Melhores 100 (Top 10) & \multicolumn{2}{|c|}{20} & \multicolumn{2}{|c|}{38} \\
\hline Melhores 25 (Top 25) & \multirow{2}{*}{\multicolumn{2}{|c|}{$\begin{array}{l}4 \\
3\end{array}$}} & \multirow{2}{*}{\multicolumn{2}{|c|}{$\begin{array}{l}9 \\
5\end{array}$}} \\
\hline Melhores 10 (Top 10) & & & & \\
\hline
\end{tabular}


Tabela 4 - Distribuição das 100 melhores vacas antes e após ajustamento para heterogeneidade da variância conforme a classe de desvio-padrão para produção de leite do rebanho

Table 4 - Distribution of cows of the top 100 before and after adjustment for heterogeneous variance by milk yield standard deviation class

\begin{tabular}{|c|c|c|}
\hline $\begin{array}{l}\text { Classes de desvio- } \\
\text { padrão (kg) } \\
\text { Standard deviation } \\
\text { class (kg) }\end{array}$ & $\begin{array}{l}\text { Antes de } \\
\text { ajustamento } \\
\text { Before } \\
\text { adjustment } \\
(\%)\end{array}$ & $\begin{array}{c}\text { Após } \\
\text { ajustamento } \\
\text { After } \\
\text { adjustment } \\
(\%)\end{array}$ \\
\hline$\geq 1310$ & 2 & 16 \\
\hline $1311-1640$ & 23 & 38 \\
\hline $1641-1970$ & 40 & 30 \\
\hline$>1970$ & 35 & 16 \\
\hline
\end{tabular}

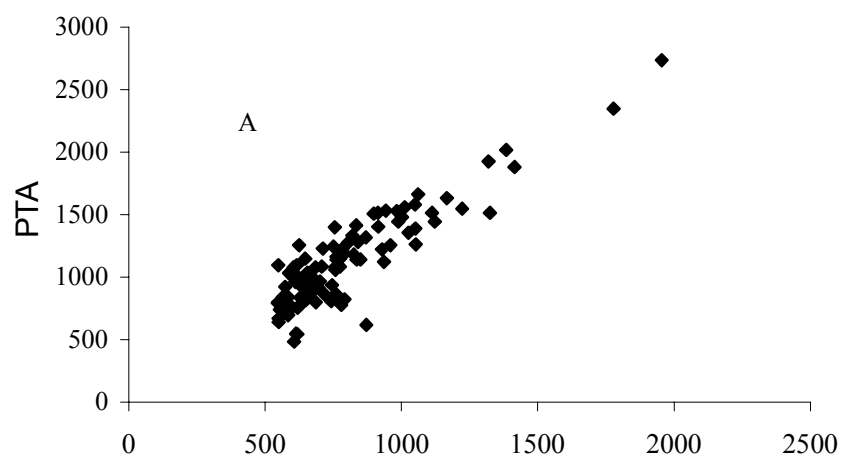

PTAC

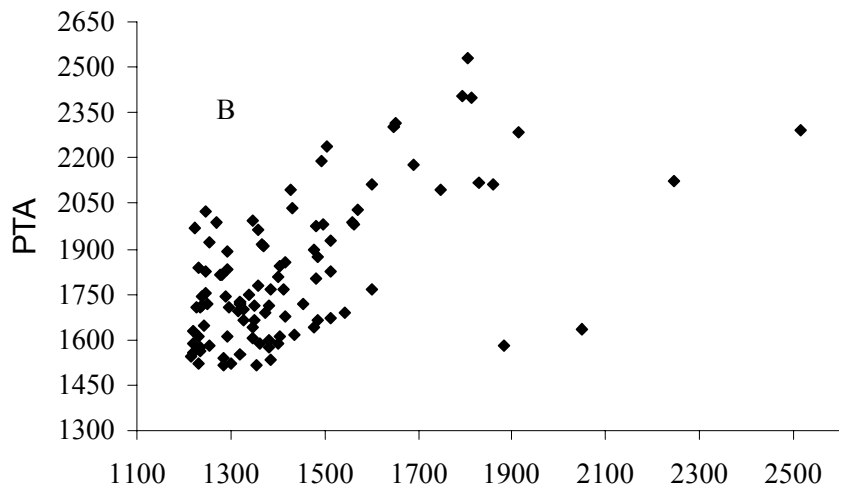

PTAC

Figura 1 - Dispersão dos 100 melhores animais conforme seus PTAs para leite antes (PTA) e após (PTAC) ajustamento para heterogeneidade da variância dentro da subclasse rebanho-ano: A) Melhores 100 touros e B) Melhores 100 vacas.

Figure 1 - Plot of top 100 animals for PTA milk before (PTA) and after (PTAC) adjustment for heterogeneous variance within herd-year subclass: A) top 100 sires and B) top 100 cows.

R. Bras. Zootec., v.31, n.1, p.369-375, 2002 (suplemento) sujeita a vícios devido a diferenças de variabilidade dentro do rebanho.

O efeito do ajustamento sobre a tendência genética para a população de vacas é ilustrado na Figura 2. No período de 1983 a 1997, o valor estimado da tendência aumentou de aproximadamente $12 \mathrm{~kg} / \mathrm{ano}$ com o ajustamento para heterogeneidade.

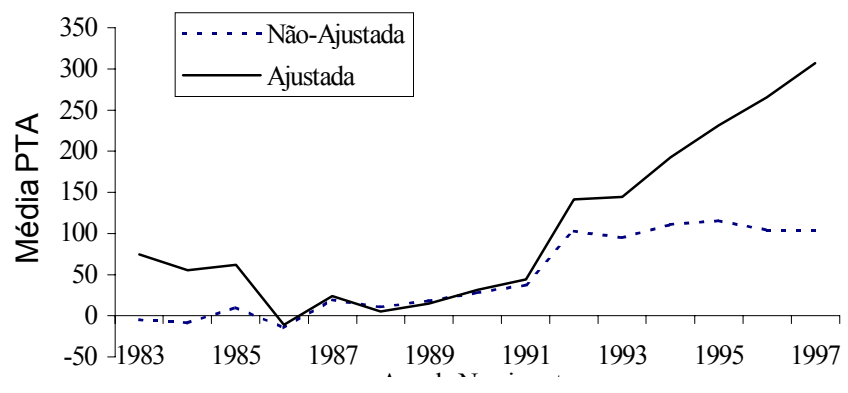

Ano de nascimento

Figura 2 - Médias das PTAs das vacas para produção de leite em avaliação sem ajustamento e com ajustamento para heterogeneidade da variância dentro de rebanho-ano por ano de nascimento.

Figure 2 - Averages of PTA milk yield for cows before and after adjustment for heterogeneity of variance within herdyear subclass by birth year.

\section{Conclusões}

Com o ajustamento para heterogeneidade de variância usado, constataram-se melhorias na avaliação genética. Mudanças de rebanhos que contribuíram com vacas elite para aqueles com variância média deverão possibilitar avaliações mais precisas e justas para identificação das melhores vacas. O procedimento deverá ser adotado nas futuras avaliações genéticas para produção de leite, na raça Holandesa, no Estado de Minas Gerais, até que novas pesquisas sugiram outro mais adequado.

\section{Literatura Citada}

BOLDMAN, K.G.; FREEMAN, A.E. Adjustment for heterogeneity of variances by herd production level in dairy cow and sire evaluation. Journal of Dairy Science, v.73, n.2, p.503-512, 1990.

BOLDMAN, K.G., L.A.; KRIESE, L., D.; VAN VLECK, C.P. et al. A manual for use of MTDFREML. A set of programs to obtain estimates of variances and covariances [DRAFT]. Beltsville: U.S. Department of Agriculture, Agricultural Research Service, 1995. 
DONG, M.C.; MAO, I.L. Heterogeneity of (co)variance and heritability in different levels of intra-herd milk production variance and of herd average. Journal of Dairy Science, v.73, n.3, p.843-851, 1990.

DURÃES, M.C.; FREITAS, A.F.; TEIXEIRA, N.M. et al. Fatores de ajustamento para a produção de leite e gordura na raça Holandesa para rebanhos do Estado de Minas Gerais. Revista Brasileira de Zootecnia, v.28, n.6, p.1231-1238, 1999.

GIANOLA, D. On selection criteria and estimation of parameters when the variance is heterogeneous. Theoretical and Applied Genetics, v.72, p.671-677, 1986.

HENDERSON, C.R. Applications of linear models in animal breeding. Guelph: University Guelph, 1984. 439p.

HILL, W.G. On selection among groups with heterogeneous variance. Animal Production, v.39, n.3, p.473-477, 1984.

MEUWISSEN, T.H.E.; Van DER WERF, J.H.J. Impact of heterogeneous within herd variances on dairy cattle breeding schemes. Livestock Production Science, v.33, n.1, p.31-41, 1993.

MIRANDE, S.L.; Van VLECK, L.D. Trends in genetic and phenotypic variances for milk production. Journal of Dairy Science, v.68, n.9, p.2278-2286, 1985.

RAMOS, A.A.; VALENCIA, E.F.T.; WECHSLER, F.S. et al. Heterogeneidade da variância das características de produção de bovinos da raça Holandesa no trópico. I. estratificação por nível de produção de rebanho. In: REUNIÃO ANUAL DA SOCIEDADE BRASILEIRA DE ZOOTECNIA, 33., 1996, Fortaleza. Anais... Fortaleza: Sociedade Brasileira de Zootecnia, 1996. p.71-73.

ROBERT-GRANIÉ, C.; BONAÏTI, B.; BOICHARD, D. et al. Accounting for variance heterogeneity in French dairy cattle genetic evaluation. Livestock Production Science, v.60, n.2, p.343-357, 1999.

TORRES, T.R.; BERGMAN, J.A.G.; COSTA, C.N. et al. Ajustamento para heterogeneidade de variância para produção de leite entre rebanhos da raça Holandesa no Brasil. Revista Brasileira de Zootecnia, v.28, n.2, p.295-303, 1999.
VALÊNCIA, E.F.T.; RAMOS, A.A.; WECHSLER, F.S. et al. Heterogeneidade da variância das características de produção de bovinos da raça Holandesa no trópico. II. Estratificação por nível de produção de vacas. In: REUNIÃO ANUAL DA SOCIEDADE BRASILEIRA DE ZOOTECNIA, 33., 1996, Fortaleza. Anais... Fortaleza: Sociedade Brasileira de Zootecnia, 1996. p.68-70.

WEIGEL, K.A.; GIANOLA, D. Estimation of heterogeneous within-herd variance components using empirical Bayes methods: a simulation study. Journal of Dairy Science, v.75, n.10, p.2824-2833. 1992.

WEIGEL, K.A.; GIANOLA, D.; YANDELL, B.S. et al. Identification of factors causing heterogeneous within-herd variance components using a structural model for variances. Journal of Dairy Science, v.76, n.5, p.1466-1478, 1993.

WIGGANS, G.H.; Van RADEN, P.M. Method and effect of adjustment for heterogeneous variance. Journal of Dairy Science, v.74, n.2, p.4350-4357, 1991.

WINKELMAN, A.; SCHAEFFER, L.R. Effect of heterogeneity of variance on dairy sire evaluation. Journal of Dairy Science, v.71, n.11, p.3033-3039, 1988.

Van DER WERF, J.H.J.; MEUWISSEN, T.H.E.; JONG, G. Effects of correction for heterogeneity of variance on bias and accuracy of breeding value estimation for Dutch dairy cattle. Journal of Dairy Science, v.77, n.10, p.3174-3184, 1994. 